\title{
ANALYSIS ON DIGITAL AUTOMATIC ASSEMBLY TECHNOLOGY UNDER ULTRASONIC TRANSDUCER
}

\author{
Xinyu Feng ${ }^{1}$, Xijing Zhu ${ }^{1}$, Xiangmeng Li $^{1}$ \\ ${ }^{1}$ School of Mechanical Engineering, North University of China, Taiyuan 030051, China. \\ Email:fe032@sina.com
}

\begin{abstract}
To study the digital automatic assembly technology, firstly, based on the working principle of ultrasonic transducer, a sandwich piezoelectric ultrasonic transducer with resonance frequency of $20 \mathrm{kHz}$ is designed by selecting component materials and calculating corresponding parameters, and the digital automatic assembly technology of sandwich piezoelectric ultrasonic transducer is proposed. Then, for the control system that is more important, the Programmable Logic Controller (PLC) control system is designed from hardware and software and PLC control system is designed through WinCC Flexible programming. Finally, digital automatic assembly technology is used to conduct primary and secondary assembly of sandwich piezoelectric ultrasonic transducers to test the performance of ultrasonic transducers in resonance frequency, impedance, and capacitance values under different assembly voltages and assembly times. The results show that when the assembly voltage increases gradually, the resonant frequency of the ultrasonic transducer also increases; when the assembly voltage is $5.5 \mathrm{~V}$ and the resonance frequency is stable at about $19.6 \mathrm{kHz}$, the impedance value presents a decreasing trend on the whole; when assembly voltage is $6.5 \mathrm{~V}$ and the impedance value is stable at around $8.5 \Omega$, assembly voltage has little influence on ultrasonic transducer capacitance; through the comparison of assembly times, it is found that the resonance frequency of secondary assembly is lower than that of primary assembly, the impedance and capacitance are higher and the performance of ultrasonic transducer decreases obviously after the second assembly. Therefore, the digital automatic assembly technology can be realized through ultrasonic transducer, which provides experimental basis and ideas for the development of digital automatic assembly technology.
\end{abstract}

Keywords: Sandwich Piezoelectric Ultrasonic Transducer; Digital Automation; Assembly Technology; Resonance Frequency; Assembly Voltage.

\section{Introduction}

With the progress and development of science and technology, ultrasonic technology, due to its good direction, strong penetration ability, easy to obtain in a concentrated manner, and long propagation distance, is currently a more in-depth research field in the field of acoustics, and has been applied and developed in industry, agriculture, medicine, military, and other fields [1]. For example, in the medical field, ultrasonic technology can analyze and monitor the human body, and clinical ultrasound diagnosis technologies such as intracavitary ultrasound and angiography have emerged, which is an important medical detection method [2]. In industry, ultrasonic waves can be used to clean and process electronic parts, instruments and equipment [3].

At the same time, ultrasonic equipment came into being. Among them, the ultrasonic transducer is the most important part of the ultrasonic equipment and reflects the performance of the whole ultrasonic equipment $[4,5]$.
Ultrasonic transducers convert ultrasonic electrical signals into ultrasonic mechanical signals. The commonly used ultrasonic transducers are magnetostrictive and piezoelectric transducers.

The design principle of the magnetostrictive transducer is the magnetic field effect of the magnetic material, and piezoelectric transducers are the most widely used ultrasonic transducers, which can stabilize the vibration in the high frequency range with high stability and low cost $[6,7]$.

Relevant studies have found that the quality and performance of piezoelectric ultrasonic transducers are closely related to their processing quality and assembly quality. Thus, for ultrasonic transducers, it needs to pay attention to the manufacturing and assembly techniques [8].

However, the conventional ultrasonic transducer assembly technology will reduce the conversion efficiency of the transducer, which is easy to cause such problems as heating and fracture of piezoelectric ceramic.

Therefore, it is necessary to study the digital automatic assembly technology based on ultrasonic 
transducer to realize the automatic assembly of ultrasonic transducer [9].

The core of PLC is the central processing unit, which combines computer and automatic control technology, has high reliability, convenient programming, is easy to diagnose and repair faults, and has been widely used in various control fields $[10,11]$.

Based on this, a sandwich piezoelectric ultrasonic transducer with a resonant frequency of $20 \mathrm{kHz}$ is designed by selecting the component materials of the piezoelectric ultrasonic transducer and calculating the corresponding parameters. The digital automatic assembly technology of sandwich piezoelectric ultrasonic transducer is proposed. At the same time, for the more important control system in the scheme, the PLC control system is designed from hardware and software. PLC control system is designed by WinCC Flexible programming to realize digital automatic assembly of ultrasonic transducer.

\section{Methodology \\ 2.1 Design of piezoelectric ultrasonic transducer}

Ultrasonic transducer converts the input electrical power into mechanical power (that is, ultrasonic) and then transmits out, and its own power consumption is less.

The components of ultrasonic transducer include shell, matching layer, piezoelectric disc transducer, backing, lead cable, and cymbal array receiver, which are shown in Figure 1 [12].

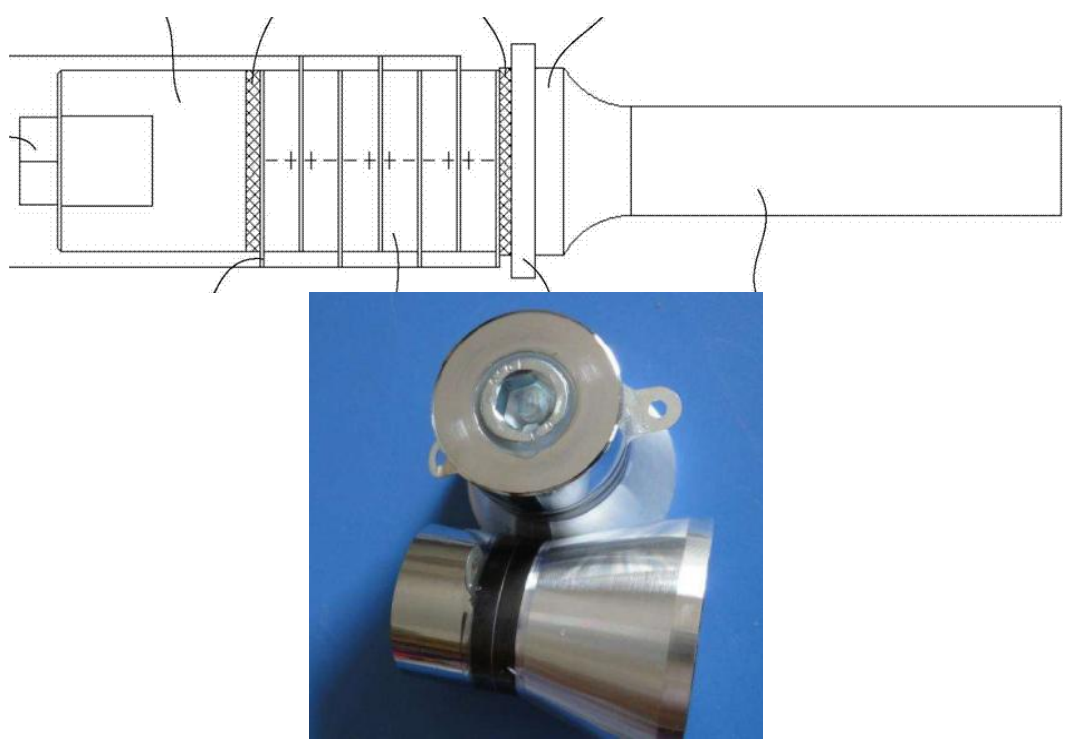

Figure 1: The ultrasonic transducer.

During ultrasonic processing, piezoelectric halfwavelength transducers with operating frequency of 20-50 Hz are often used.

When monolithic piezoelectric ceramics are used, the thickness is $40-100 \mathrm{~mm}$, the processing is difficult, and the mechanical and chemical quality factors can't meet the expected requirements with high energy consumption and low frequency, which doesn't have high use value.

At the same time, the amplitude of monolithic piezoelectric ceramic transducer is small in resonance state.
Therefore, the sandwich piezoelectric ultrasonic transducer is used in this study, and the nonpiezoelectric material with high quality factor value and small expansion coefficient is used at both ends.

Sandwich piezoelectric ultrasonic transducer is mainly composed of front and rear end cover and piezoelectric ceramic plate, which is partly assembled by bolt.

In this study, a sandwich piezoelectric ultrasonic transducer with a resonant frequency of $20 \mathrm{kHz}$ is designed. The material selection and corresponding parameters are shown in Table 1.

Table 1. Material selection and corresponding parameters of sandwich piezoelectric ultrasonic transducer

\begin{tabular}{|l|l|l|}
\hline Components & Material selection & Parameters \\
\hline $\begin{array}{l}\text { Piezoelectric } \\
\text { ceramic piece }\end{array}$ & PZT-8 & $\begin{array}{l}\text { 4 pieces, diameter } 55 \mathrm{~mm} \text {, central hole 23mm, thickness 7 mm } \\
\rho_{0}=7.8 \mathrm{~g} / \mathrm{cm}^{3}, C=3.4 \times 10^{6} \mathrm{~mm} / \mathrm{s}, K_{33}=0.63\end{array}$ \\
\hline $\begin{array}{l}\text { Front and rear } \\
\text { end covers }\end{array}$ & $\begin{array}{l}\text { Aluminum alloy } \\
\text { LY12 }\end{array}$ & Diameter $55 \mathrm{~mm}, \rho_{1}=2.6 \mathrm{~g} / \mathrm{cm}^{3}, C_{1}=C_{2}=5.2 \times 10^{6} \mathrm{~mm} / \mathrm{s}$ \\
\hline $\begin{array}{l}\text { Metal electrode } \\
\text { plate }\end{array}$ & Red copper & Diameter $55 \mathrm{~mm}, L_{3}=0.2 \mathrm{~mm}$ \\
\hline
\end{tabular}


If $\mathrm{L}_{1}$ is the length of the rear end cover of the sandwich piezoelectric ultrasonic transducer, $\mathrm{L}_{2}$ is the length of the front-end cover, when considering the thickness of piezoelectric crystal pile, the resonant frequency equation of sandwich piezoelectric ultrasonic transducer is as follows.

$$
\tan K_{0} L_{01} \cdot \tan K_{1} L_{1}=\left(1-K_{33}^{2}\right) \cdot \frac{Z_{0}}{Z_{1}}
$$

In Eq. 1, L01represents the distance from the nodal surface to the rear end cover, which is 18.9 $\mathrm{mm}$.

$$
\begin{aligned}
& K_{0}=\frac{\omega}{C_{0}}=\frac{2 \pi f}{C_{0}}=4.05 \times 10^{-2}, \\
& K_{1}=\frac{\omega}{C_{1}}=\frac{2 \pi f}{C_{1}}=2.46 \times 10^{-2}, \\
& \frac{Z_{0}}{Z_{1}}=\frac{\rho_{0} C_{0}}{\rho_{1} C_{1}}=1.71 .
\end{aligned}
$$

The length of the rear end cover can be obtained by substituting it into Eq. 1 , that is $L_{1}=37.31 \mathrm{~mm}$.

Similarly, Eq. 2 is obtained.

$$
\tan K_{0} L_{02} \cdot \tan K_{1} L_{2}=\left(1-K_{33}^{2}\right) \cdot \frac{Z_{0}}{Z_{1}}
$$

$\mathrm{L}_{02}$ represents the distance from nodal surface to the front rear cover, which is $6.3 \mathrm{~mm}$. the length of the frontend cover can be obtained by substituting it into Eq. 2, that is $L_{2}=55.15 \mathrm{~mm}$.

\subsection{Design of digital automatic assembly based on sandwich piezoelectric ultrasonic transducer}

The digital automatic assembly technology of sandwich piezoelectric ultrasonic transducer can stabilize the torque of ultrasonic transducer and make the piezoelectric ceramic plates neatly arranged. The following conditions shall be met in the design: I. In the process of tightening, it is necessary to ensure that there is no relative sliding movement between piezoelectric ceramic plates, copper plates, and other components; II. during the tightening process, it needs to keep the torque stable and meet the installation requirements; III. it is advisable to set stop protection device, when there is a problem, the machine can be shut down in time; IV. the mechanical structure of ultrasonic transducer can meet the design requirements of rigidity and strength.

The components of digital automatic assembly machine based on sandwich piezoelectric ultrasonic transducer include motor, three-jaw chuck, socket wrench, mechanical transmission structure, digital signal acquisition, and processing system and PLC controller. The three-jaw chuck is used to clamp the sandwich piezoelectric ultrasonic transducer during the working process, and the presence of the motor causes the pressure plate to drop and the ultrasonic transducer to be compressed.

The second motor exists to rotate the socket wrench and tighten the nut of the ultrasonic transducer. Due to the piezoelectric effect in the process of tightening, thus generating the charge, the digital signal acquisition system is used to measure the voltage value at both ends of the ultrasonic transducer, and the PLC controller is used to compare the voltage value.

When the voltage value is equal to the initial set value, the PLC control motor shuts down and runs to complete the assembly of the sandwich piezoelectric ultrasonic transducer.

The main process of using the sandwich piezoelectric ultrasonic transducer automatic assembly machine includes the following steps.

Step 1: the sandwich piezoelectric ultrasonic transducer is put on the three-jaw chuck. The nut of the ultrasonic transducer is aligned with the socket wrench so that it is on the same vertical line. Then the ultrasonic transducer is fixed and the measuring clips are clamped on the positive and negative electrodes respectively.

Step 2: the second servo motor is turned on, the press plate is dropped, and the ultrasonic transducer is pressed down.

Step 3: the first servo motor is turned on, the socket wrench is turned, and the ultrasonic transducer is tightened. When the voltage rises to the set value, it needs to turn off the first servo motor, turn on the second servo motor, and raise the pressure plate.

In the process of voltage acquisition, AT89S51 is used to achieve the control effect, and AD574 is used for A/D conversion. In the system circuit, the AD574 works synchronously, with a conversion accuracy of 12 bits and a measurement conversion time of up to $25 \mu \mathrm{s}$. AD574 has high integration, small circuit size, low power consumption, good shielding, and requires fewer external components. AT89S51 is a CMOS8-bit microcontroller with low energy consumption and strong performance of, which can be flexibly applied in a variety of occasions with high cost performance.

The chip Max485 is used to connect the interface circuit of AT89S51 and PC. The chip is an RS-485 chip, the power supply is $+5 \mathrm{~V}$, the communication mode is half duplex, and the level can be converted.

The protocol communication with PLC is realized by RS-485 cable. In the preparation of the MCU program, its workflow is to initialize the serial port, set communication parameters and working mode, and write the command to receive the reply data. The PLC receives the command, responds, and returns the reply frame.

The data frame subroutine flow of the communication between MCU and PLC is shown in Figure 2. 


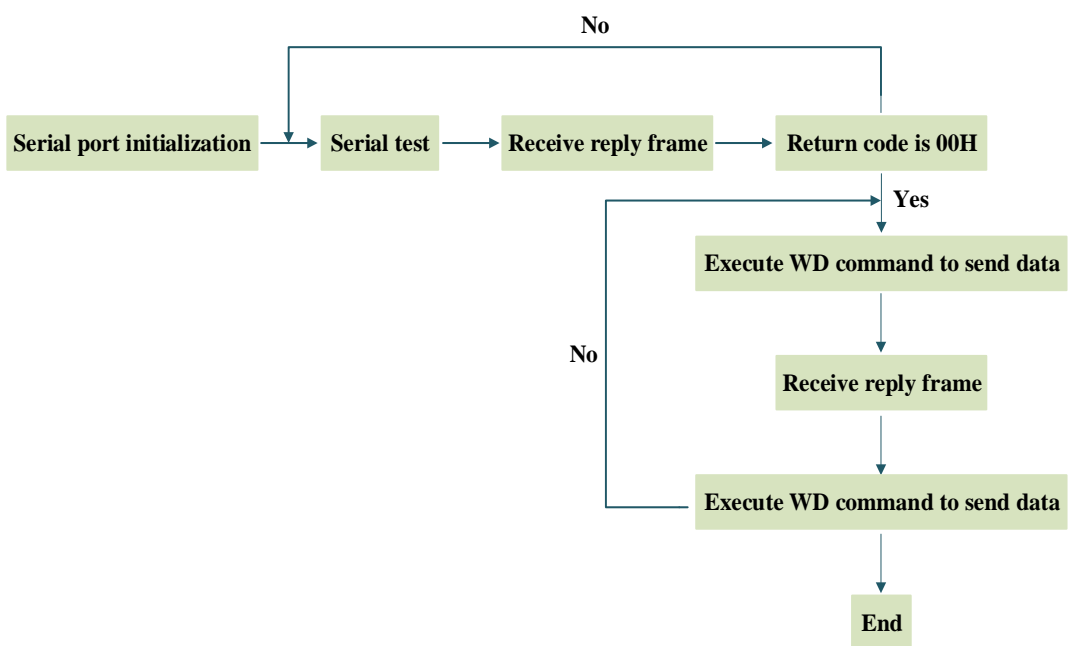

Figure 2: Data frame subroutine flow of single chip microcomputer and PLC communication.

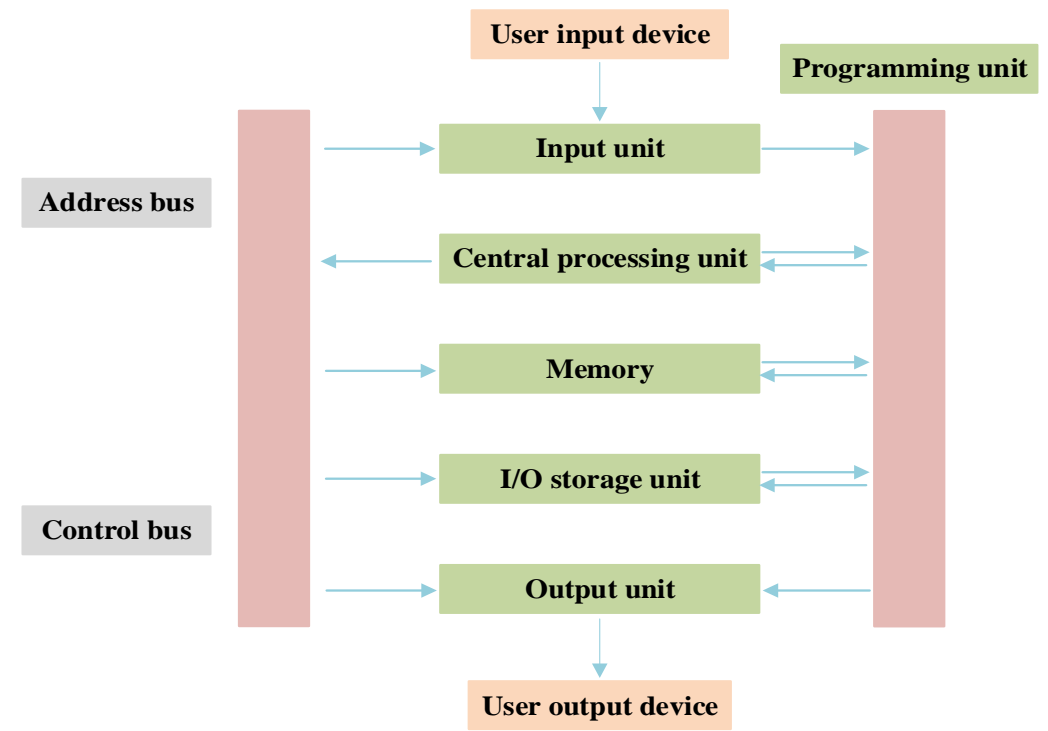

Figure 3: PLC structure.

The control system of sandwich piezoelectric ultrasonic transducer automatic assembly machine is designed by PLC control program. The PLC uses a typical computer structure to transform the input and output of a particular algorithm and to physically implement it. The input and output are to process the information, and the physical realization is to apply the input of PLC to the industrial field. Its structure is shown in Figure 3.

The PLC control system is designed mainly through hardware and software two parts to control objects. The design steps of the system are to determine the I/O device, select the proper PLC type, distribute I/O addresses, design PLC program, carry out PLC adjustment and test, and operate the system, as shown in Figure 4.

The hardware of the control system is mainly composed of upper computer, PLC controller, motor driver, and data acquisition system, as shown in Figure 5. In this study, the S7-200 series CPU 224XP
CN PLC control system is selected to design the heart type piezoelectric ultrasonic transducer automatic assembly machine. Dc power supply is $24 \mathrm{~V}$ with wide communication capacity and internal clock components.

The main function of the stepper motor is to control and rotate at a fixed angle without accumulated errors, and the application field is wide, such as open loop control. The stepper motor driver is required to drive the stepper motor and convert the pulse signal sent by the control system into the angular displacement of the stepper motor.

Therefore, by controlling the frequency of the pulse signal, accurate speed regulation of the motor can be realized. By controlling the number of pulses, the motor can be precisely located. In this study, a three-phase hybrid sinusoidal subdivision driver of HB308SN is selected, with a power supply of $220 \mathrm{~V}$ and a current output of $8 \mathrm{~A}$. 


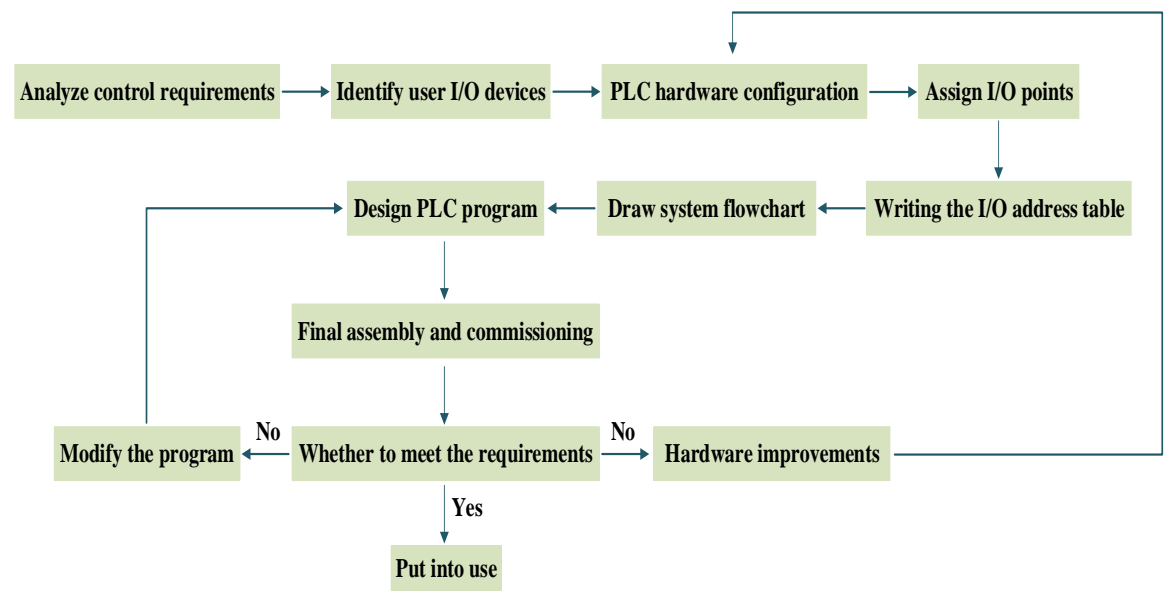

Figure 4: Design flow of PLC control system.

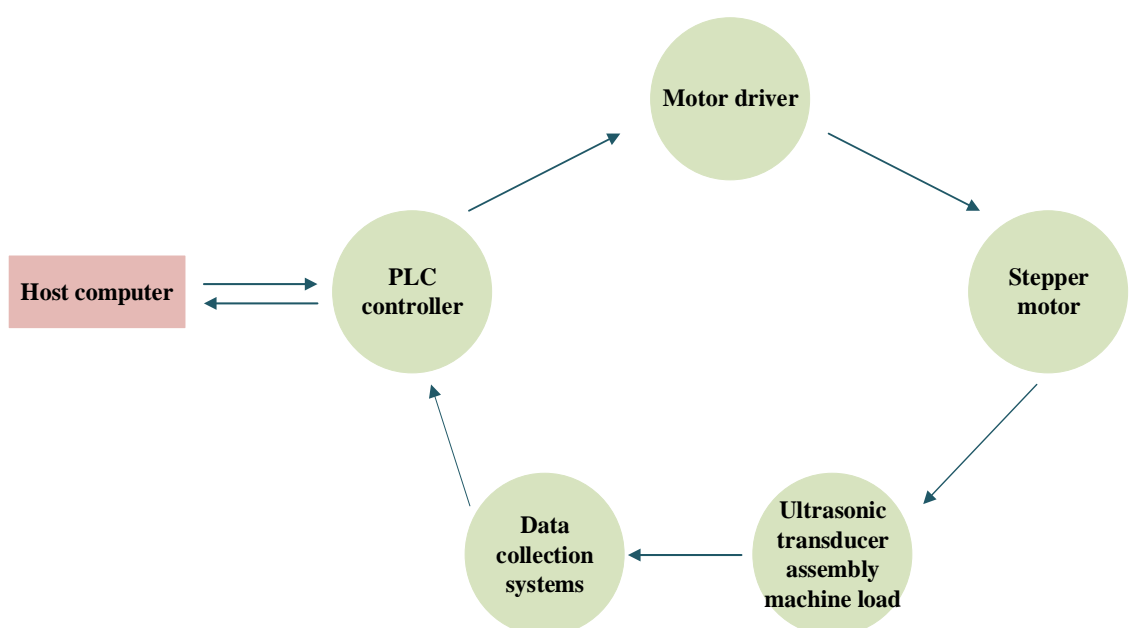

Figure 5: The hardware structure of control system.

WinCC Flexible is adopted to program the control and monitoring system of sandwich piezoelectric ultrasonic transducer automatic assembly machine. WinCC Flexible, based on Windows platform, is a computer-based human-machine interface configuration software, which can realize the visual operation of the system and has rich functions. WinCC Flexible internally integrates Active X control, which can integrate human interface into TCP/IP network [13].

In this research system, Siemens MP370 12-inch touch screen is used to realize man-machine interface.

The HMI device can access the process value stored by I/O module in the PLC through "variables", and the steps to design the HMI operation and monitoring system are as follows: first, the new HMI project is built in WinCC Flexible; then, communication is established between PLC and HMI and between computer and HMI equipment, respectively, and the process variables are defined; finally, the configuration screen is drawn and the elements and variables in the screen are connected for control and monitoring.

\subsection{Experimental steps of digital automatic assembly of sandwich piezoelectric ultrasonic transducer}

The preassembly steps of sandwich piezoelectric ultrasonic transducer are as follows.

I. the bolt is screwed into the ultrasonic head, and then the insulation sleeve is inserted into the bolt;

II. keeping the axis of the four piezoelectric ceramic plates and the two copper plates in the same vertical line, the positive and negative electrodes of the piezoelectric ceramic are connected to the positive and negative electrodes by two copper plates;

III. the piezoelectric ceramic piece and copper piece are put into the bolt and covered outside the insulator;

IV. the reflector is slipped onto the bolt and screwed on the nut. At this time, the sandwich piezoelectric ultrasonic transducer preassembly work is basically completed.

The assembly process of ultrasonic transducer in one assembly is as follows.

I. first, the preload value of the system is set to 1.0 ; 
II. the compression motor power supply is started and the motor power supply is tightened;

III. the parameters are set. The down pulse period of compression is set as 500, the up pulse period of compression is set as 300 , the number of up pulses is set as 60,000 , the tightening positive pulse period is set as 1500, the number of unscrewed pulses for the first time is set as 100, and the number of unscrewed pulses for the second time is set as 100;

IV. the control mode of the compression motor and the control mode of the tightening motor are set as automatic state respectively;

$\mathrm{V}$. the ultrasonic transducer is aligned with the socket wrench;

VI. the positive and negative electrodes of the measuring clip are connected with the positive and negative electrodes of the transducer copper plate and are clamped;

VII. the knob is tightened and the transducer is clamped;

VIII. after the discharge, the ultrasonic transducer is loosened, the switch is turned off, and the transducer is removed.

The assembly process of the ultrasonic transducer in the secondary assembly (that is, it has been assembled once and reassembled after removal) is basically the same as that in the first assembly.

The difference lies in canceling step IV in an assembly and proceeding step V -step VII in turn. Then, the compression motor control mode and tightening motor control mode are set to manual mode respectively. The pressing manual is set as downward, and the manual is tightened as unscrew; the transducer nut is loosened, and the pressing manual is set as upward, and a distance is left between the pressure plate and the transducer.

Then, the compression motor control mode and tightening motor control mode are set to automatic state respectively, and the above step VIIIis performed.

In this study, the TH2818 automatic component analyzer is selected to measure the assembled ultrasonic transducer, which has a wide measuring range and high accuracy.

The capacitance is measured by VICTOR 6013 capacitance meter, which is small in volume and operated by manual range.

\section{Results and Discussion}

\subsection{Test results of resonance frequency, impedance, and capacitance}

The sandwich piezoelectric ultrasonic transducer is assembled using digital automatic assembly technology.

The assembly voltage value of the automatic assembly machine is set as $3-8 \mathrm{~V}$, and the resonant frequency, impedance, and capacitance values of the sandwich piezoelectric ultrasonic transducer are measured, as shown in Figure 6.

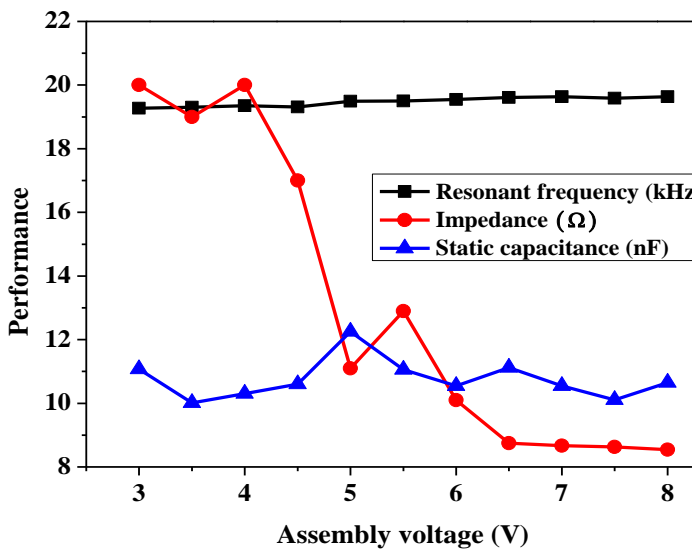

Figure 6: The resonance frequency, impedance, and capacitance values of sandwich piezoelectric ultrasonic transducers at different rated voltages.

As the rated voltage of the assembly increases, the resonant frequency increases gradually, but the increase range is small. When the assembly voltage is $5.5 \mathrm{~V}$, the resonant frequency basically does not change much, and the value is about $19.6 \mathrm{khz}$, which is basically consistent with the initial design of the $20-\mathrm{kHz}$ ultrasonic transducer. As the assembly voltage increases, the impedance value generally decreases. When assembly voltage is $6.5 \mathrm{~V}$, the impedance value basically remains unchanged, and its value is approximately $8.5 \Omega$.

When the assembly voltage increases gradually, the capacitance of ultrasonic transducer increases and decreases, with an average value of about $10.75 \mathrm{nF}$, indicating that the capacitance is not affected by the rated assembly voltage.

\subsection{Performance comparison of sandwich piezoelectric ultrasonic transducers between primary assembly and secondary assembly}

Figure 7 shows the performance comparison of sandwich piezoelectric ultrasonic transducers with primary assembly and secondary assembly, where the assembly voltage is set as $7 \mathrm{~V}$.

The resonance frequency of the secondary assembly is lower than that of the primary assembly, while the impedance and capacitance are both higher than that of the primary assembly, indicating that the performance of ultrasonic transducer decreases obviously after the second assembly.

The reason is that the properties of piezoelectric ceramics change during a single assembly. Therefore, in the process of digital automatic assembly of piezoelectric ultrasonic transducer, the secondary assembly of ultrasonic transducer can't be carried out. 


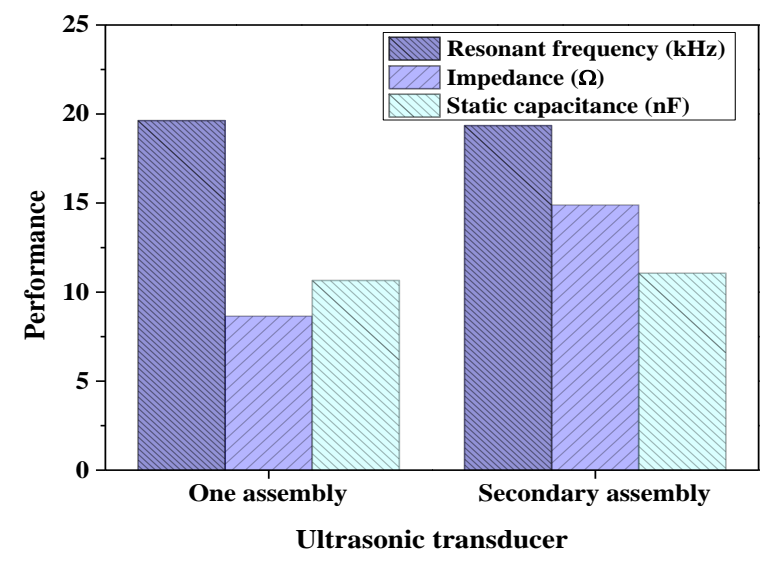

Figure 7: Performance comparison of sandwich piezoelectric ultrasonic transducers between primary assembly and secondary assembly.

\section{Conclusions}

In order to study the digital automatic assembly technology, a sandwich piezoelectric ultrasonic transducer with resonance frequency of $20 \mathrm{kHz}$ is designed. The digital automatic assembly technology design scheme of sandwich piezoelectric ultrasonic transducer is proposed, the sandwich piezoelectric ultrasonic transducer is assembled once and twice, and the resonance frequency, impedance, and capacitance of ultrasonic transducers with different assembly voltages and assembly times are tested.

When the assembly voltage gradually increases, the resonant frequency of the ultrasonic transducer also gradually increases, and the impedance value generally presents a decreasing trend. However, the capacitance of ultrasonic transducer is not greatly affected by the assembly voltage, and the performance of ultrasonic transducer obviously decreases after the second assembly.

This study realizes the digital automatic assembly technology through ultrasonic transducer, which provides a certain foundation for the development of digital automatic assembly technology.

However, there are still some defects in the digital automatic assembly technology of sandwich piezoelectric ultrasonic transducer.

In this study, the automatic assembly technology of ultrasonic transducer is studied at close range, and its feasibility can't be guaranteed at a remote distance.

Therefore, in the future research work, it needs to consider to set up the wireless remote monitoring equipment to achieve the remote operation of digital automatic assembly technology.

\section{References}

[1] V. Krishna, F.Sammartino, A. Rezai. "A review of the current therapies, challenges, and future directions of transcranial focused ultrasound technology: advances in diagnosis and
treatment."JAMA neurology, vol.75, no.2, pp. 246254, 2018.

[2] F.TovoliF, V. Cantisani, C. Schiavone, et al. "What Future for Ultrasound in Medicine?"Ultraschall in der Medizin-European Journal of Ultrasound,vol.39, no. 01, pp. 7-10, 2018.

[3] C. D. Goldsmith, Q. V. Vuong, C. E. Stathopoulos, et al. "Ultrasound increases the aqueous extraction of phenolic compounds with high antioxidant activity from olive pomace."LWT, vol. 89,pp. 284290, 2018.

[4] S.Nakamoto, R. Sano, K. Kanda, et al. "Multilayer $\mathrm{Pb} \quad(\mathrm{Zr}, \mathrm{Ti}) \mathrm{O} 3$ thin films for ultrasonic transducer."Electronics and Communications in Japan, vol.101, no.4, pp. 63-68, 2018.

[5] M. O. Khyam, S. S. Ge, X. Li, et al. "Pseudoorthogonal chirp-based multiple ultrasonic transducer positioning."IEEE Sensors Journal, vol.17, no.12, pp. 3832-3843, 2017.

[6] Y. Li, W. Huang, B. Wang, et al. "High-Frequency Output Characteristics of Giant Magnetostrictive Transducer." IEEE Transactions on Magnetics, vol.55, no.6, pp. 1-, 20195.

[7] Z.Briqech, A. R. Sebak, T. A. Denidni. "Low-cost wideband mm-Wave phased array using the piezoelectric transducer for 5G applications."IEEE Transactions on Antennas and Propagation, vol.65, no.12, pp. 6403-6412, 2017.

[8] W. H. Liew, K. Yao, S. Chen, et al. "Piezoelectric nanotube array for broadband high-frequency ultrasonic transducer."IEEE transactions on ultrasonics, ferroelectrics, and frequency control, vol.65, no.3, pp. 457-464, 2017.

[9] S.Guo, S. Chen, L. Zhang, et al. "Plastic strain determination with nonlinear ultrasonic waves using in situ integrated piezoelectric ultrasonic transducers."IEEE transactions on ultrasonics, ferroelectrics, and frequency control, vol. 65, no.1, pp. 95-101, 2017.

[10] R. G. Baldovino, E. P. Dadios. "A Fuzzy Proportional-Derivative (PD) Algorithm for Programmable Logic Controller (PLC)." Advanced Science Letters, vol.23, no.11, pp. 11400-11403, 2017.

[11] P. Cronin, F. S. Hosseini, C. Yang. "A Low Overhead Solution to Resilient Assembly Lines Built from Legacy Controllers."IEEE Embedded Systems Letters, vol.10, no.3, pp. 103-106, 2018.

[12] S. M. Ji, J. H. Sung, C. Y. Park, et al. "Phasecanceled backing structure for lightweight ultrasonic transducer."Sensors and Actuators A: Physical, vol.260, pp. 161-168, 2017.

[13] Q. Song, J. Xiao, L. Wen, et al. "Temperature Field During Automated Fiber Placement for Thermoplastic Composite."Journal of Materials Engineering, vol.46, no.1, pp. 83-91, 2017. 